\title{
Nonlinear coherent Thomson scattering from relativistic electron sheets as a means to produce isolated ultrabright attosecond $\mathrm{x}$-ray pulses
}

\author{
H.-C. Wu (武慧春), ${ }^{1} *$ J. Meyer-ter-Vehn, ${ }^{2}$ B. M. Hegelich, ${ }^{1}$ and J. C. Fernández ${ }^{1}$ \\ ${ }^{1}$ Los Alamos National Laboratory, Los Alamos, New Mexico 87545, USA \\ ${ }^{2}$ Max-Planck-Institut für Quantenoptik, D-85748 Garching, Germany
}

(Received 23 January 2011; published 14 July 2011)

\begin{abstract}
A new way to generate intense attosecond $\mathrm{x}$-ray pulses is discussed. It relies on coherent Thomson scattering (CTS) from relativistic electron sheets. A double layer technique is used to generate planar soliddensity sheets of monochromatic high- $\gamma$ electrons with zero transverse momentum such that coherently backscattered light is frequency upshifted by factors up to $4 \gamma^{2}$. Here previous work [H.-C. Wu et al., Phys. Rev. Lett. 104, 234801 (2010)] is extended to the regime of high-intensity probe light with normalized amplitude $a_{0}>1$ leading to nonlinear CTS effects such as pulse contraction and steepening. The results are derived both by particle-in-cell (PIC) simulation in a boosted frame and by analytic theory. PIC simulation shows that powerful $\mathrm{x}$-ray pulses $(1 \mathrm{keV},<10$ attosecond, and $>10$ gigawatt) can be generated. They call for experimental verification. Required prerequisites such as manufacture of nanometer-thick target foils is ready and ultrahigh contrast laser pulses should be within reach in the near future.
\end{abstract}

DOI: 10.1103/PhysRevSTAB.14.070702

PACS numbers: 41.75.Jv, 41.60.- m, 42.55.Vc, 52.59.Ye

\section{INTRODUCTION}

Because of broad applications in many fields of science and technology, there are strong motivations to develop bright and coherent $\mathrm{x}$-ray sources. These sources include free-electron lasers, $\mathrm{x}$-ray lasers, and high harmonics from both atomic interaction in gases and relativistic laser interaction in plasmas. Presently, the leading x-ray source is the free-electron laser, which can output powerful $(>10$ gigawatt) coherent light pulses in the extreme ultraviolet as well as in the soft and hard x-ray regime $[1,2]$ and tens of femtoseconds long. Such high-quality sources are opening up a new era for extreme ultraviolet and x-ray interaction with matter and provide unprecedented opportunities for research in condensed matter physics, high energy density physics, and molecular imaging for biology.

Bright and coherent $\mathrm{x}$-ray sources can also be obtained by means of coherent Thomson scattering (CTS) from dense electron layers, flying with a relativistic factor $\gamma_{x}=$ $1 / \sqrt{1-\beta_{x}^{2}}$. Here $\beta_{x}=v_{x} / c$ is the velocity of the plane layer in normal direction. Counterpropagating probe light is then reflected and frequency upshifted by the relativistic Doppler factor, which is $\left(1+\beta_{x}\right) /\left(1-\beta_{x}\right) \approx 4 \gamma_{x}^{2}$ for $\gamma_{x} \gg 1$ [3]. We refer to such an electron layer as a relativistic electron sheet (RES).

One way to produce RES is to drive cold nonlinear plasma waves to the point of wave breaking. The density

\footnotetext{
*Corresponding author.

hcwu@lanl.gov or huichunwu1@gmail.com

Published by the American Physical Society under the terms of the Creative Commons Attribution 3.0 License. Further distribution of this work must maintain attribution to the author(s) and the published article's title, journal citation, and DOI.
}

profile then develops diverging spikes that move with high $\gamma_{x}$ factors [4,5]. RES with a higher $\gamma_{x}$ factor and electron density can be achieved by accelerating electrons from ultrathin solid foils by few-cycle laser pulses with sharply rising fronts. This regime has been described by Kulagin in a number of papers (see e.g. [6]). In order to push all electrons out of the foil, the normalized field $a_{0, d}=$ $e E_{0} / m c \omega_{0}$ of the drive laser must be larger than the normalized field arising from charge separation, $\epsilon=$ $\left(n_{e} / n_{c}\right) k_{0} L$. Here $\omega_{0}=c k_{0}$ is the angular frequency of the driving laser pulse, $n_{e}$ is electron density assuming complete ionization by the laser front, $L$ is the initial thickness of the foil, and $n_{c}=\varepsilon_{0} m \omega_{0}^{2} / e^{2}$ is the critical density.

The use of these relativistic electron sheets for coherent Thomson backscattering has been studied in previous papers $[7,8]$, and it became clear that there are some serious drawbacks. First, transverse electron motion inherent to direct acceleration in the laser field leads to $\gamma_{x}$ much smaller than the total $\gamma$. The Doppler shift factor turns out to be $4 \gamma_{x}^{2} \approx 2 \gamma$, just the square root of the full factor $4 \gamma^{2}$. Second, $\gamma$ depends on time and thus the reflected pulse is chirped and has a broad spectrum. Third, the transverse motion degrades planar stability and reduces the available reflective surface.

Recently, significant progress has been made by a scheme proposed in [9]. This scheme circumvents all the drawbacks mentioned above. It is illustrated in Fig. 1. The key element is an additional foil located behind the RESproducing foil that is just thick enough to reflect the drive laser pulse, but allows the relativistic electrons to go through almost unperturbed. Before passing the reflector foil, the electrons now interact with both the drive and the reflected drive pulse. As a result, their transverse 


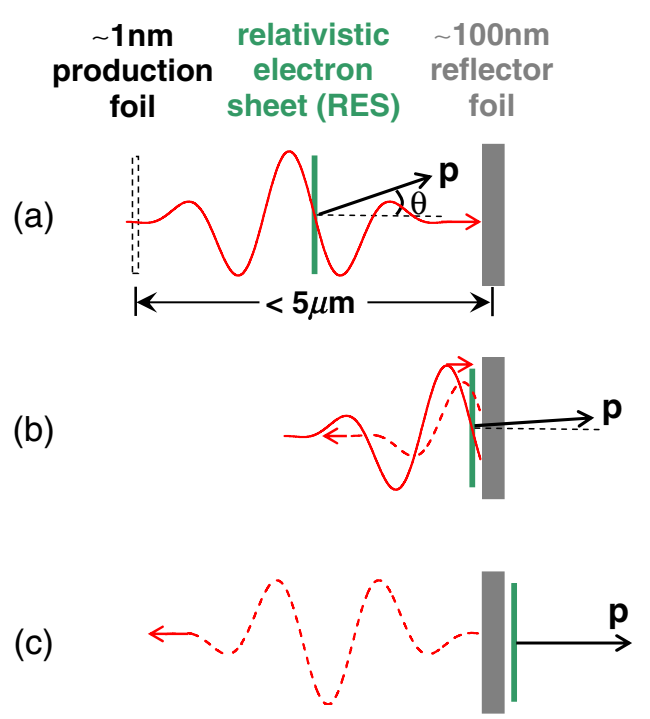

FIG. 1. Scheme for producing the relativistic electron sheet (RES). (a) An intense, normally incident, few-cycle laser pulse (red) has ionized the ultrathin (and therefore transparent) production foil and has expelled all electrons. They form the RES (green), surfing on the laser pulse. Electron momenta $\mathbf{p}$ are tilted relative to the laser propagation axis by an angle $\theta$ due to the transverse component of the laser field. (b) The laser pulse is reflected by the reflector foil (grey) and interacts a second time with the RES. The reflector foil is just thick enough to fully reflect the driving laser pulse, but to let the relativistic electrons pass almost unperturbed. (c) The RES emerges into field-free space behind the reflector. Because of conservation of canonical momentum, electrons now move exactly in normal direction with zero transverse momentum.

momentum is completely canceled, while their longitudinal momentum is almost unchanged. The RES emerges from the reflector foil with a high and fixed relativistic factor $\left(\gamma_{x}=\gamma\right)$. It may backscatter counterpropagating probe light with a full Doppler shift factor $4 \gamma^{2}$, and the scattered signal has a narrow spectrum. These features are documented in Fig. 2 in terms of a one-dimensional (1D) particle-in-cell (PIC) simulation. Also the area of the RES is approximately equal to the laser spot size. It should be understood that the lifetime of the high-density RES is rather short ( $\sim$ tens of femtosecond) due to Coulomb expansion, but it is long enough to produce ultrashort high-intensity x-ray pulses.

To date, analysis and simulation have been done in the linear CTS regime, i.e., for probe light amplitudes $a_{0}^{2} \ll 1$. In this regime, the amplitude of the scattered signal is proportional to $a_{0}$. Of course, it is of high interest to get more powerful $\mathrm{x}$-ray output and therefore to raise the intensity of the probe light up to the relativistic regime of $a_{0} \geq 1$. This is what is studied in the present paper on the basis of PIC simulation and analytic theory. The Doppler factor by which the RES of given $\gamma$ can upshift the frequency of the backscattered probe light is $4 \gamma^{2} /\left(1+a_{0}^{2}\right)$ (for circular polarization), which it decreases for $a_{0} \geq 1$
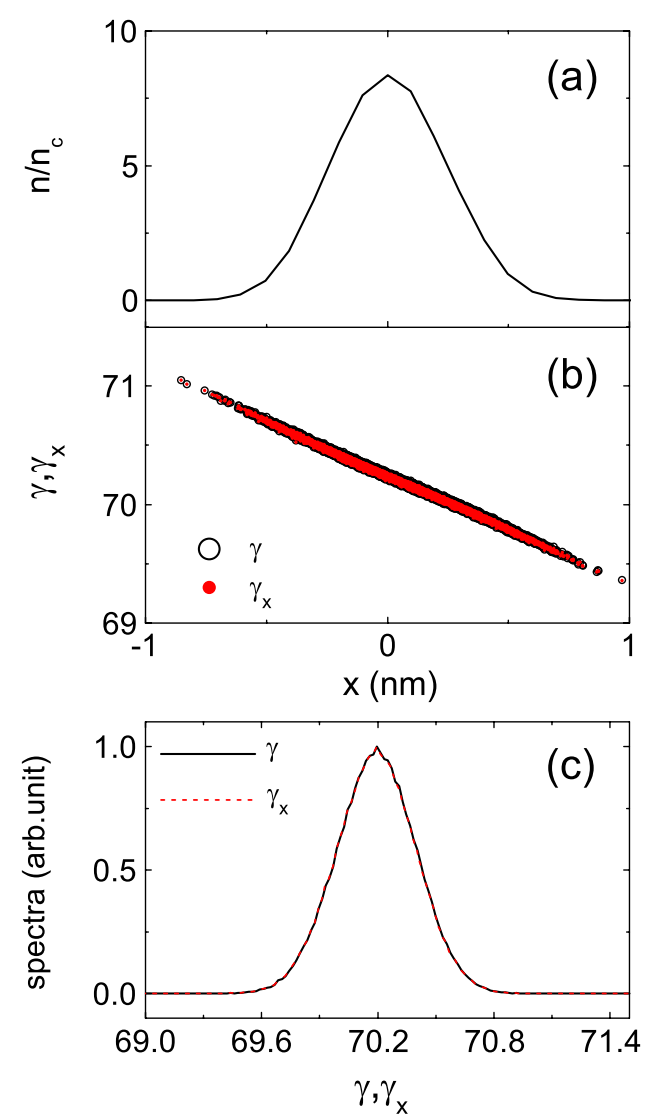

FIG. 2. PIC results for a laser-driven RES obtained for the configuration of Fig. 1: (a) electron density profile; (b) $\gamma$ and $\gamma_{x}$ plotted versus propagation direction $x$; (c) electron energy spectrum. The results correspond to Fig. 1(c), i.e., to the time when the RES has just emerged from the reflector foil. Simulation parameters are given in Sec. II A.

due to recoil of sheet electrons. CTS sensitivity to sheet thickness and nonlinear effects such as output saturation and self-shortening phenomenon are discussed. The simulations show that gigawatt $\mathrm{keV}$ attosecond $\mathrm{x}$-ray pulses can be generated.

Before the end of this section, we would like to discuss the current state of art and potential challenges for realizing our scheme in experiments. This is helpful to attract more experimental interests. Apparently, graphene is a good candidate for the RES production foil. Nowadays, freestanding graphene has a size of hundreds of microns [10], and tens of inches of graphene on some substrate materials has been successfully fabricated [11]. Moreover, the nanomeshed graphene technique [12] allows varying electron areal density of the production foil. About two-foil spacing, the piezoelectricity technique [13] can easily control micron-scale separation with a precision of tens of $\mathrm{nm}$. So, target techniques for our purpose are mature. The big challenge is high-contrast terawatt few-cycle lasers. The optical parametric chirped-pulse amplification (OPCPA) technique can support broadband few-cycle light amplification [14]. At present, the OPCPA technique 
produced sub-three-cycle (8 fs) $16 \mathrm{TW}$ laser pulse [15]. The upgrade to $5 \mathrm{fs} / 20 \mathrm{TW}$ or $100 \mathrm{TW}$ is ongoing [16]. The planned European laser project ELI [17] aims to produce $5 \mathrm{~J} / 5$ fs petawatt laser pulse. These laser systems are suitable for RES generation. Of course, pulse contrast is a big issue and has to exceed $10^{-12}$, because any significant prepulse may dissociate the graphene before the arrival of the main pulse. Although it is demanding and challenging, clean few-cycle or even single-cycle intense lasers should be reachable in the near future.

The paper is organized as follows. In Sec. II, RES generation is reviewed in terms of 1D PIC simulation and then CTS of a probe pulse is simulated in the RES rest frame, applying boosted PIC technique. In Sec. III, 1D analytic theory of nonlinear CTS is derived, and in Sec. IV, results of theory and simulation are discussed.

\section{SIMULATIONS OF RES GENERATION AND NONLINEAR CTS}

In this section, we present 1D PIC simulations of nonlinear CTS from a RES using JPIC [18]. Though RES generation is not the central issue of this paper (in this respect the reader should refer to the previous publication [9]), we start here from PIC results providing RES particle and energy distributions. They are obtained for particular target and drive laser parameters and are then used for the CTS simulation in a boosted frame.

\section{A. Uniform RES generation}

A double layer target (see Fig. 1) is used to generate a uniform RES with $\gamma_{x}=\gamma$ [9]. The first foil is referred to as the sheet production foil from which the relativistic electron layer is pushed out. It is chosen with a thickness of $L=0.5 \mathrm{~nm}$ and an electron density of $n_{0}=10 n_{c}$. It is thinner than the skin depth and therefore transparent to the driving laser pulse. Choosing a linearly polarized single-cycle wave with temporal shape $a_{0, d} \sin \left(\omega_{0} t\right)$ for $0 \leq \omega_{0} t \leq 2 \pi$ and amplitude $a_{0, d}=40$, all foil electrons are ionized and taken along with the wave forming the RES, while, on the short time scale, the heavy ions are left behind. The $\mathbf{v} \times \mathbf{B}$ force of the light, exceeding the space charge force, drives all electrons to almost the same high $\gamma$ value over a short distance.

The second target foil, the reflector foil, is designed to completely reflect the driving laser pulse. It is chosen with a thickness of $L_{r}=80 \mathrm{~nm}$, a density of $n_{0, r}=800 n_{c}$, and is placed $1.88 \lambda_{0}$ behind the production foil. Here $\lambda_{0}=$ $800 \mathrm{~nm}$ is the laser wavelength. In the 1D PIC simulation, we haven chosen 8000 cells $/ \lambda_{0}$ and 40000 macroparticles per cell. An initial temperature of $10 \mathrm{eV}$ is taken for the production sheet to mimic ionization, while the reflector foil is initialized as cold plasma with zero temperature.

Figure 2 shows density profile, phase space, and energy spectrum at the moment depicted in Fig. 1(c) when the RES has just emerged from the rear side of the reflector foil. In Fig. 2(a), it is seen that the central density of the RES has just dropped a little bit to $8.4 n_{c}$ from its initial value of $10 n_{c}$ in the production foil and has expanded from $0.5 \mathrm{~nm}$ to a FWHM of $0.565 \mathrm{~nm}$. The RES energy spectrum is given in Fig. 2(c) sharply peaked at $\gamma=70.2$. The energy of each electron is also depicted in Fig. 2(b) versus propagation coordinate $x$. The RES internal energy chirp and spread $(\sim 0.66 \%)$ is due to the nonuniform acceleration of the layer; leftmost electrons of the RES in Figs. 2(a) and 2(b) experience the field earlier and have gained slightly higher energy.

Actually, we have plotted both $\gamma$ and $\gamma_{x}$ values on top of each other in Figs. 2(b) and 2(c) in order to feature the important fact that, after having passed the reflector foil, all RES electrons are free of transverse momentum and that therefore $\gamma=\gamma_{x}$. Indeed, it is seen that the numerically obtained values of $\gamma$ and $\gamma_{x}$ coincide very accurately for each electron and cannot be distinguished in the plots. It should be recalled [9] that $\gamma$ and $\gamma_{x}$ differ vastly in front of the reflector foil, where RES electrons experience the driving laser field and have significant transverse momenta. The cancellation of transverse momentum when passing the reflector foil is due to conservation of canonical momentum, $\mathbf{p}_{\perp}-\mathbf{a}=$ const, that holds for planar symmetry, $\mathbf{p}_{\perp}$ is normalized by $m c$. In the field-free region behind the reflector $(\mathbf{a} \equiv 0)$ transverse electron momenta turn back to initial zero values. In fact, the simple configuration described here allows for perfect directional conversion of laser beams into electron beams. This does not contradict the Lawson-Woodward criterium [19], stating that direct conversion of light into particle beams is not possible in free space. It is the interaction with walls that enables the conversion (see also discussion in [20]). 2D PIC simulation is also carried out with the same parameters as Fig. 2. Without any substantial difference from 1D PIC results, a uniform RES free of transverse motion is generated after the reflector with a transverse size of about laser spot size.

The cancellation of transverse momentum is of central importance in the following sections of this paper, where the RES is used for coherent Thomson backscattering. It enables CTS with full Doppler shift of $4 \gamma^{2}$ in the linear regime for probe light with amplitude $a_{0} \ll 1$. For larger amplitudes $a_{0}>1$, however, when the probe beam modifies electron motion of the RES significantly, the maximum Doppler shift is again reduced in a nonlinear way depending on $a_{0}$, and also the amplitude of the scattered signal saturates. This is discussed in the following sections.

\section{B. Nonlinear CTS}

The high Doppler shift factors of up to $4 \gamma^{2}$ make it difficult to time resolve the high-frequency CTS signal in PIC simulations. In order to perform fast and high-precision simulations, we therefore employ the ultrafast boosted PIC technique [21]. All the information of the 
electrons shown in Fig. 2 is transformed into the RES rest frame (see Sec. III B and Appendix A), in which the PIC simulation is performed. In this boosted frame, the CTS signal is resolved with 200 time steps per cycle.

Figure 3 shows both temporal profile and spectrum of the CTS signal obtained with the sample RES shown in Fig. 2. Here, we use a circular polarized probe pulse colliding head-on with the RES and having an envelope $a_{0} \sin ^{2}(\pi t / T)$, where $a_{0}=5$ and $T=10 \lambda_{0} / c$. The scattered signal is also circular polarized and has a peak amplitude $a_{\mathrm{cts}}=0.2$, which corresponds to an intensity of $I_{\text {cts }}=1.7 \times 10^{17} \mathrm{~W} / \mathrm{cm}^{2}$. For a radiating area of $10 \mu \mathrm{m}^{2}$, the peak power would be $17 \mathrm{GW}$. The duration of the CTS pulse is about 5 as. The central frequency is $790 \omega_{0}$, which corresponds to an x-ray wavelength of $1 \mathrm{~nm}$ $(1.2 \mathrm{keV})$. There are about $10^{9}$ photons within this $\mathrm{x}$-ray pulse. Focused to the diffraction-limited area of $1 \mathrm{~nm}^{2}$, the intensity will rise to $\sim 10^{24} \mathrm{~W} / \mathrm{cm}^{2}$. The Doppler shift factor is on the order of $4 \gamma^{2} / a_{0}^{2}$. Also, the x-ray pulse contains only four light cycles different from the ten cycles of the incident pulse. These are consequences of nonlinear interaction that will be explained by analytic theory in the following sections.

Let us add here a short remark on three-dimensional RES evolution. One should consider the possibility that the relativistically intense probe laser pulse may split and destroy the RES in the transverse directions during the CTS process. We can estimate the transverse oscillation amplitude of electrons in the scattering field as

$$
Y=Y^{\prime}=\frac{a_{0}}{k_{0}^{\prime}}=\frac{a_{0}}{k_{0} \sqrt{D_{n l}}} \approx \frac{a_{0}^{2}}{4 \pi \gamma_{0}} \lambda_{0}
$$
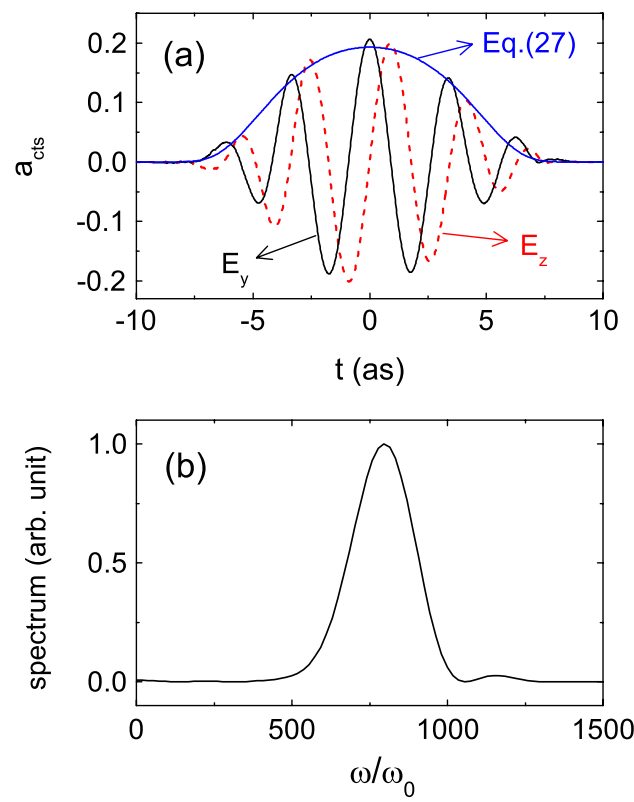

FIG. 3. Nonlinear CTS from RES in Fig. 2. (a) Temporal shape of CTS signal and theoretical calculation from Eq. (27). (b) Spectrum of CTS signal. which is typically much smaller than the laser wavelength $\lambda_{0}$. Here $Y^{\prime}$ is the oscillation amplitude in the boosted frame and $D_{n l}$ is the nonlinear Doppler shift factor [see Eq. (12)]. Electrons remain local in the transverse direction. For the example in Fig. 2 , one has $Y \approx 0.03 \lambda_{0}$. Thus, the electrons will not experience the nonuniform transverse ponderomotive force on the scale of the laser spot size. Therefore the RES should be quite stable for nonlinear CTS.

\section{THEORY OF NONLINEAR CTS}

In this section, we derive the 1D nonlinear theory for CTS at relativistic intensities. We Lorentz transform to the rest frame of the RES, derive the CTS signal amplitude in this frame, and then transform back to the lab frame [8]. The Lorentz transformation to the boosted frame is derived in Appendix A. Figure 4 illustrates schematically positions of electrons and waves before interaction and during Thomson scattering in both lab and boosted frames.

\section{A. RES dynamics and Doppler shift factor}

CTS depends on the collective effect of a large number of electrons performing almost identical dynamics. Here we impose planar symmetry of the RES moving in normal direction. The scattered signal is then well described in a single electron model, keeping in mind that the full angular complexity of nonlinear Thomson scattering of single electrons (see the classical paper by Sarachik and Schappert [22]) cancels out due to planar symmetry and destructive interference.

We consider an incident electron with energy $\gamma_{0}=$ $\sqrt{1+p_{0}^{2}}$ and momentum $p_{0}$ colliding with a counterpro-

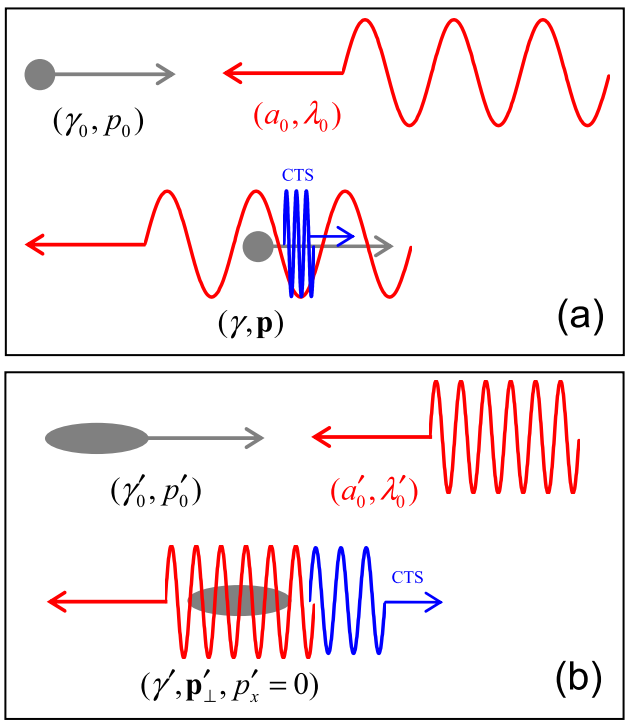

FIG. 4. Schematic drawings for electrons $\left(\gamma_{0}, p_{0}\right)$, incident waves $\left(a_{0} . \lambda_{0}\right)$, and backscattered waves (CTS) before interaction and during CTS in both lab (a) and boosted (b) frames. 
pagating plane circular polarized laser pulse. The laser pulse has the vector potential $\mathbf{a}=e \mathbf{A} / m c=a_{0} f(\tau) \times$ $\left[\hat{y} \cos \left(\omega_{0} \tau\right), \hat{z} \sin \left(\omega_{0} \tau\right)\right]$, where $f(\tau)$ represents the shape function of the pulse with the rising front starting from $\left.f\right|_{\tau=0}=0$ and having the maximum at $\left.f\right|_{\tau \rightarrow \infty}=1$ with $\tau=t+x / c$. For instance, the shape function can be taken as $f(\tau)=\tanh (\tau / T)$, where $T$ measures the rising time. The goal is to calculate CTS amplitude and transfer efficiency at the time when $|\mathbf{a}|=a_{0}$ or $f=1$.

For the interaction geometry defined in Fig. 4(a), there are two constants of motion for electrons in the scattering field [23]:

$$
\begin{aligned}
& \mathbf{p}_{\perp}-\mathbf{a}=\text { const, } \\
& \gamma+p_{x}=\text { const. }
\end{aligned}
$$

Considering the initial conditions $\left.\mathbf{p}_{\perp}\right|_{\tau=0}=\left.\mathbf{a}\right|_{\tau=0}=0$ and $\left.\left(\gamma+p_{x}\right)\right|_{\tau=0}=\gamma_{0}+p_{0}$, the solutions are

$$
\begin{gathered}
\mathbf{p}_{\perp}=\mathbf{a}, \\
p_{x}=\frac{\left(\gamma_{0}+p_{0}\right)^{2}-\left(1+a^{2}\right)}{2\left(\gamma_{0}+p_{0}\right)}, \\
\gamma=\frac{\left(\gamma_{0}+p_{0}\right)^{2}+\left(1+a^{2}\right)}{2\left(\gamma_{0}+p_{0}\right)} .
\end{gathered}
$$

When the laser field reaches the maximum $a=a_{0}$, they are

$$
\begin{gathered}
\left.\mathbf{p}_{\perp}\right|_{a=a_{0}}=\mathbf{a}_{0}, \\
\left.p_{x}\right|_{a=a_{0}}=\frac{\left(\gamma_{0}+p_{0}\right)^{2}-\left(1+a_{0}^{2}\right)}{2\left(\gamma_{0}+p_{0}\right)}, \\
\left.\gamma\right|_{a=a_{0}}=\frac{\left(\gamma_{0}+p_{0}\right)^{2}+\left(1+a_{0}^{2}\right)}{2\left(\gamma_{0}+p_{0}\right)}
\end{gathered}
$$

The longitudinal velocity and the related gamma factor are (with subscript $a=a_{0}$ simplified as $a_{0}$ )

$$
\begin{aligned}
& \left.\beta_{x}\right|_{a_{0}}=\frac{\left(\gamma_{0}+p_{0}\right)^{2}-\left(1+a_{0}^{2}\right)}{\left(\gamma_{0}+p_{0}\right)^{2}+\left(1+a_{0}^{2}\right)}, \\
& \left.\gamma_{x}\right|_{a_{0}}=\frac{\left(\gamma_{0}+p_{0}\right)^{2}+\left(1+a_{0}^{2}\right)}{2\left(\gamma_{0}+p_{0}\right) \sqrt{1+a_{0}^{2}}} .
\end{aligned}
$$

So, the nonlinear Doppler shift factor is

$$
D_{n l}=\frac{1+\left.\beta_{x}\right|_{a_{0}}}{1-\left.\beta_{x}\right|_{a_{0}}}=\frac{\gamma_{0}^{2}\left(1+\beta_{0}\right)^{2}}{1+a_{0}^{2}},
$$

which depends on the laser amplitude $a_{0}$. It reduces to the linear result $D_{l}=\gamma_{0}^{2}\left(1+\beta_{0}\right)^{2}$ for $a_{0}^{2} \rightarrow 0$. The CTS wavelength is $\lambda_{\text {cts }}=\lambda_{0} / D_{n l}$. Apparently, the relativistically intense probe pulse reduces the electron longitudinal velocity by means of its nonlinear ponderomotive force and thus degrades the linear Doppler shift factor $D_{l}$ by a factor $\left(1+a_{0}^{2}\right)$. For the reference results given in Fig. 3, Eq. (12) leads to $D_{n l}=758$, in reasonable agreement with the simulation result given in Fig. 3(b).

For a linear polarized pulse $a_{0} \hat{y} \cos \left(\omega_{0} \tau\right)$, the cycleaveraged nonlinear Doppler shift factor is $D_{n l}=$ $\left(\gamma_{0}+p_{0}\right)^{2} /\left(1+a_{0}^{2} / 2\right)$. Production of odd harmonics due to $2 \omega_{0}$ beating of $\beta_{x}$ will be discussed in a separate paper.

\section{B. RES in boosted frame and CTS amplitude}

Now we do the Lorentz transformation from the lab frame to a boosted frame moving with the velocity $\beta_{b}=$ $\left.\beta_{x}\right|_{a_{0}}$ [Eq. (10)], corresponding to the relativistic factor $\gamma_{b}=\left.\gamma_{x}\right|_{a_{0}}$ [Eq. (11)]. In the new frame, initial electron energy and momentum become

$$
\gamma_{0}^{\prime}=\frac{2+a_{0}^{2}}{2 \sqrt{1+a_{0}^{2}}}, \quad p_{0}^{\prime}=\frac{a_{0}^{2}}{2 \sqrt{1+a_{0}^{2}}} .
$$

Since transverse vector potential $\mathbf{a}(\tau)$ and phase $\tau$ are Lorentz invariants, we obtain from Eqs. (2) and (3)

$$
\begin{gathered}
\mathbf{p}_{\perp}^{\prime}=\mathbf{a}, \\
p_{x}^{\prime}=\frac{\left(\gamma_{0}^{\prime}+p_{0}^{\prime}\right)^{2}-\left(1+a^{2}\right)}{2\left(\gamma_{0}^{\prime}+p_{0}^{\prime}\right)}, \\
\gamma^{\prime}=\frac{\left(\gamma_{0}^{\prime}+p_{0}^{\prime}\right)^{2}+\left(1+a^{2}\right)}{2\left(\gamma_{0}^{\prime}+p_{0}^{\prime}\right)} .
\end{gathered}
$$

For $a=a_{0}$ they become

$$
\begin{gathered}
\left.\mathbf{p}_{\perp}^{\prime}\right|_{a_{0}}=\mathbf{a}_{0}, \\
\left.p_{x}^{\prime}\right|_{a_{0}}=0, \\
\left.\gamma^{\prime}\right|_{a_{0}}=\sqrt{1+a_{0}^{2}} .
\end{gathered}
$$

In the boosted frame, electrons do not move in the longitudinal direction when the laser amplitude reaches the maximum.

The RES initial density and thickness become

$$
\begin{aligned}
& n_{0}^{\prime}=n_{0} \gamma_{0}^{\prime} / \gamma_{0}, \\
& d_{0}^{\prime}=d_{0} \gamma_{0} / \gamma_{0}^{\prime},
\end{aligned}
$$

where $n_{0}$ and $d_{0}$ are peak density and characteristic thickness of the RES in the lab frame, defined such that $n(x)=$ $n_{0} S(x)$ and $\int n(x) d x=n_{0} d_{0}$, where $S(x)$ is a shape function. It is noted that $n_{0} / \gamma_{0}, \gamma_{0} d_{0}$, and $n_{0} d_{0}$ are Lorentz invariants.

During pulse rise, sheet compression occurs because of the nonuniform velocity, as expressed by Eq. (15). When $a=a_{0}$, the new density and thickness are

$$
\left.n^{\prime}\right|_{a_{0}}=\left.n_{0}^{\prime} \gamma^{\prime}\right|_{a_{0}} / \gamma_{0}^{\prime} \text {, }
$$




$$
\left.d^{\prime}\right|_{a_{0}}=d_{0}^{\prime} \gamma_{0}^{\prime} /\left.\gamma^{\prime}\right|_{a_{0}}
$$

where the compression ratio is $\left.\eta^{\prime} \equiv \gamma^{\prime}\right|_{a_{0}} / \gamma_{0}^{\prime}$ (see Appendix B). So, $\gamma d$ and $n / \gamma$ are conserved during compression.

Now we deduce the CTS amplitude. The CTS frequency is the same as $\omega^{\prime}$ of the scattering pulse. The vector potential of the CTS signal is

$$
\mathbf{A}_{\mathrm{cts}}^{\prime}=\frac{c \mu_{0}}{2} \int_{-\infty}^{\infty} d x^{\prime \prime} \int_{-\infty}^{t^{\prime}-\left|x^{\prime}-x^{\prime \prime}\right| / c} \mathbf{J}^{\prime}\left(x^{\prime}, t^{\prime}\right) d t^{\prime \prime},
$$

where the radiation current is

$$
\mathbf{J}^{\prime}\left(x^{\prime}, t^{\prime}\right)=-\frac{e^{2} \mathbf{A}^{\prime}\left(x^{\prime}, t^{\prime}\right)}{m} \frac{\left.n^{\prime}\left(x^{\prime}\right)\right|_{a_{0}}}{\left.\gamma^{\prime}\right|_{a_{0}}},
$$

with $\left.n^{\prime}\left(x^{\prime}\right)\right|_{a_{0}}=\left.n^{\prime}\right|_{a_{0}} S\left(x^{\prime}\right)$. The CTS electric field in the $y$ direction is obtained from $E_{\mathrm{cts}, y}^{\prime}=-\partial A_{\mathrm{cts}, y}^{\prime} / \partial t^{\prime}$. The electric field amplitude is given by

$$
E_{\mathrm{cts}, 0}^{\prime}=\left.E_{0}^{\prime} \frac{\left.e^{2} k^{\prime} d^{\prime}\right|_{a_{0}}}{2 \varepsilon_{0} m \omega^{\prime 2}} \frac{\left.n^{\prime}\right|_{a_{0}}}{\left.\gamma^{\prime}\right|_{a_{0}}}\right|_{F}(\xi) \mid,
$$

where $k^{\prime}=\omega^{\prime} / c$, the form factor $F(\xi)=\int_{-\infty}^{\infty} S(\chi) \times$ $\exp (i \xi \chi) d \chi, \quad \chi \equiv \chi^{\prime} /\left.d^{\prime}\right|_{a_{0}}, \quad$ and $\left.\xi \equiv 2 k^{\prime} d^{\prime}\right|_{a_{0}}$. Using relations $E_{0}^{\prime} / \omega^{\prime}=E_{0} / \omega_{0}, E_{\mathrm{cts}, 0}=E_{\mathrm{cts}, 0}^{\prime} \gamma_{b}\left(1+\beta_{b}\right), \omega^{\prime}=$ $\omega_{0} \gamma_{b}\left(1+\beta_{b}\right)$ and Eqs. (19)-(23), Eq. (26) is transformed back into the lab frame giving

$$
E_{\mathrm{cts}, 0}=\left.E_{0} \frac{\gamma_{0}\left(1+\beta_{0}\right)}{2\left(1+a_{0}^{2}\right)} \frac{n_{0} k_{0} d_{0}}{n_{c}}\right|_{F}(\xi) \mid .
$$

Here $\xi=2 \gamma_{0}^{2}\left(1+\beta_{0}\right) k_{0} d_{0} /\left(1+a_{0}^{2}\right)=2 k_{\mathrm{cts}} d_{0} /\left(1+\beta_{0}\right)$, and $k_{\text {cts }}=2 \pi / \lambda_{\text {cts }}$ is the wave number of the CTS signal. The relativistic effect causing the nonlinearity is represented by the denominator $\left(1+a_{0}^{2}\right)$ and $D_{n l}$, implicitly contained in F. As $a_{0}^{2} \rightarrow 0$, Eq. (27) approaches the linear result given in Ref. [9]. For a Gaussian profile $S(\chi)=\exp \left[-\pi\left(x / d_{0}\right)^{2}\right]=\exp \left(-\pi \chi^{2}\right)$, one has $F(\xi)=$ $\exp \left(-\xi^{2} / 4 \pi\right)$. For the example given in Fig. 2, $\gamma_{0}=$ $70.2, a_{0}=5$, and $d_{0} \simeq 0.6 \mathrm{~nm}$, Eq. (27) leads to $a_{\mathrm{cts}, 0}=e E_{\mathrm{cts}, 0} / m c \omega_{0}=0.193$, which agrees well with the simulation. The conversion efficiency of pulse energy can be found from Eq. (27) as

$$
\alpha=\frac{E_{\mathrm{cts}, 0}^{2}}{E_{0}^{2} D_{\mathrm{nl}}}=\frac{\left(n_{0} / n_{c}\right)^{2}\left(k_{0} d_{0}\right)^{2}}{4\left(1+a_{0}^{2}\right)}|F(\xi)|^{2} .
$$

Although Eq. (27) is obtained for a constant laser amplitude, it is also applicable to an ultrashort laser pulse as the one shown in Fig. 3(a), where the pulse envelope $a_{0} \sin ^{2}(\pi t / T)$ replaces $a_{0}$ in Eq. (27). The resulting CTS pulse shape agrees well with the PIC result.

\section{DISCUSSION AND CONCLUSIONS}

We first discuss the effect of layer thickness on CTS; it sets the most stringent limit on RES reflectivity and photon energy that can be achieved by CTS at sufficient levels of photon number. For a Gaussian-shaped layer $n_{0} \exp \left(-\pi x^{2} / d_{0}^{2}\right) \quad\left(\mathrm{FWHM}=0.94 d_{0}\right)$ with fixed areal density $n_{0} d_{0}$, the CTS amplitude is

$$
E_{\mathrm{cts}, 0} \propto F(\xi) \simeq \exp \left(-\pi d_{0}^{2} / \lambda_{\mathrm{cts}}^{2}\right) .
$$

It decays exponentially for increasing layer thickness (see Fig. 5). This sensitive effect is due to destructive interference of the radiation field from a RES with thickness larger than $\lambda_{\text {cts }}$. When $d_{0}=0.85 \lambda_{\text {cts }}$, the amplitude already drops to $10 \%$ of the maximum. For efficient CTS, one prefers $d_{0}<0.8 \lambda_{\text {cts }}$. For the example given in Fig. 3 , one has $d_{0}=$ $0.6 \lambda_{\text {cts }}$. This strong dependence on RES thickness explains why the reflected signal shown in Fig. 3(a) contains four light cycles only, rather than the ten cycles of the incident scattering pulse. It is because the Doppler factor $D_{n l}$ is quite large around the foot of the scattering pulse due to low laser amplitude [see Eq. (12)]. So the CTS wavelength $\lambda_{\text {cts }}$ is much shorter at the pulse foots, where intensity is low, and therefore $d_{0} / \lambda_{\text {cts }} \gg 1$. This in turn leads to a dramatic decrease in reflectivity near the pulse foot and reduces the number of cycles within the final CTS signal. It also cuts off the wings in the transverse distribution of the scattering pulse. We refer to this as the nonlinear selfshortening and self-contraction effect.

Also, the nonlinear CTS output saturates for ultrarelativistic amplitudes of the scattering pulse, $a_{0} \gg 1$. For fixed CTS wavelength $\lambda_{\text {cts }}$, implying $D_{n l}=$ const, one has

$$
E_{\mathrm{cts}, 0}=E_{\mathrm{cts}, \mathrm{sat}} \frac{a_{0}}{\sqrt{1+a_{0}^{2}}},
$$

which approaches the saturation value $e E_{\mathrm{cts}, \mathrm{sat}} / m c \omega_{0}=$ $\left.\left(n_{0} k_{0} d_{0} \sqrt{D_{n l}} / 2 n_{c}\right)\right|_{F}(\xi) \mid$ for $a_{0} \rightarrow \infty$ (see Fig. 6). The saturation can be understood from the radiating current [Eq. (25)] $J^{\prime} \propto a_{0} / \gamma_{0} \propto a_{0} / \sqrt{1+a_{0}^{2}}$, which approaches a maximum for $a_{0} \rightarrow \infty$. The relativistically increased electron mass leads to the saturation. From Fig. 6, we can see that the linear CTS regime, in which the CTS amplitude is

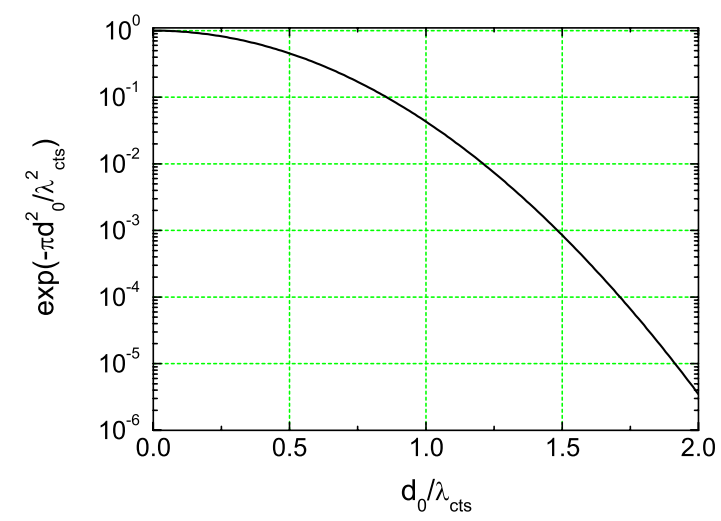

FIG. 5. RES reflectivity as a function of RES thickness $d_{0}$ divided by CTS wavelength $\lambda_{\text {cts }}$ in the lab frame. 


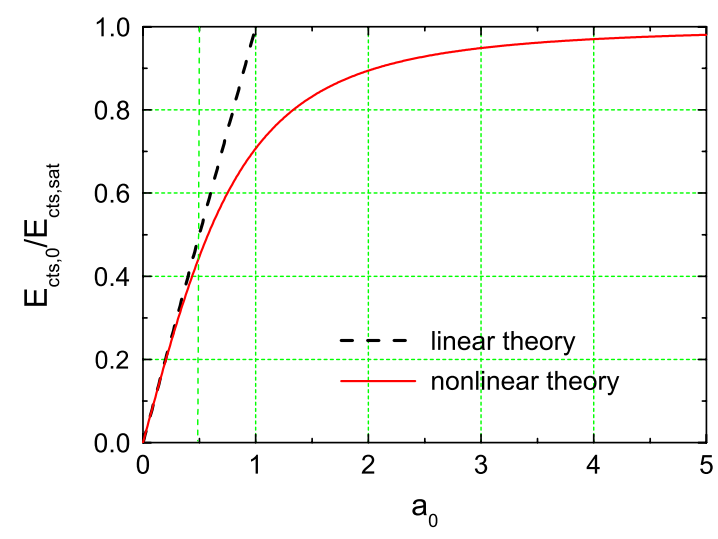

FIG. 6. Saturation effect of nonlinear CTS [Eq. (30)].

proportional to $a_{0}$, applies for $a_{0}<0.5$. At $a_{0}=2$, the CTS amplitude already amounts to $90 \%$ of the saturation level.

So we conclude that raising the amplitude of the probe pulse to relativistic levels of $a_{0}>1$ will not help to increase the intensity of the scattered pulse and that the energy of the scattered photons even decreases according to the nonlinear Doppler shift factor of $4 \gamma^{2} /\left(1+a_{0}^{2}\right)$. Nevertheless, we think these nonlinear features will become of interest in experiments and even applications. Since RES generation requires ultrahigh drive intensities to overcome the space charge field when separating electrons from ions, this implies that the resulting RES $\gamma$ values tend to be higher than useful for CTS because of $\lambda_{\text {cts }} \ll d_{0}$ and corresponding decay of the scattered amplitude, as we have just discussed. In this situation, lowering the Doppler shift by shifting the probe pulse to relativistic intensities may help to produce sufficient numbers of scattered photons. We expect that playing with the amplitudes of the drive laser pulse $a_{0, d}$ determining $\gamma$ and that of the probe laser pulse $a_{0}$, the nonlinear effects arising for $a_{0}>1$ will allow for optimizing and spatial shaping of the $\mathrm{x}$-ray output.

\section{ACKNOWLEDGMENTS}

This work is supported by the Laboratory Directed Research and Development (LDRD) Program No. 20110341ER at the Los Alamos National Laboratory. H.-C. Wu wishes to thank Dr. Rahul Shah for improving the text. J. Meyer-ter-Vehn is grateful for the support from the Munich center for Advanced Photonics (MAP) and the Association EURATOM-Max-PlanckInstitute for Plasma Physics.

\section{APPENDIX A: BOOSTED PIC SIMULATION}

Lorentz boosted PIC technique [21] is embedded in JPIC [18] to simulate the CTS process. One needs to make Lorentz transformation from the lab frame to the boosted frame as discussed in Sec. III B. The sheet energy, density, and thickness in the new frame are given by Eqs. (13), (20), and (21). The laser wavelength is given by
TABLE I. Simulation parameters of Fig. 2 in both lab and boosted frames.

\begin{tabular}{lrcccc}
\hline \hline Frame & $\gamma_{0}, \gamma_{0}^{\prime}$ & $\frac{n_{0}}{n_{c}}, \frac{n_{0}^{\prime}}{n_{c}^{\prime}}$ & $\frac{d_{0}}{\lambda_{0}}, \frac{d_{0}^{\prime}}{\lambda^{\prime}}$ & $a_{0}$ & $\lambda_{0}, \lambda^{\prime}$ \\
\hline Lab & 70.2 & 8.4 & $7.5 \times 10^{-4}$ & 5 & $800 \mathrm{~nm}$ \\
Boosted & 2.6 & $4.2 \times 10^{-4}$ & 0.55 & 5 & $29 \mathrm{~nm}$ \\
\hline \hline & $\lambda^{\prime}=\lambda_{0} \frac{\sqrt{1+a_{0}^{2}}}{\gamma_{0}+p_{0}}$, & (A1)
\end{tabular}

which defines a critical density

$$
n_{c}^{\prime}=n_{c} \frac{\left(\gamma_{0}+p_{0}\right)^{2}}{1+a_{0}^{2}} .
$$

New normalized density and thickness are

$$
\begin{gathered}
\frac{n_{0}^{\prime}}{n_{c}^{\prime}}=\frac{n_{0}}{n_{c}} \frac{\left(2+a_{0}^{2}\right) \sqrt{1+a_{0}^{2}}}{2 \gamma_{0}\left(\gamma_{0}+p_{0}\right)^{2}}, \\
\frac{d_{0}^{\prime}}{\lambda^{\prime}}=\frac{d_{0}}{\lambda_{0}} \frac{2 \gamma_{0}\left(\gamma_{0}+p_{0}\right)}{2+a_{0}^{2}} .
\end{gathered}
$$

The normalized laser amplitude is $a_{0}^{\prime}=a_{0}$. Also pulse shape and cycles within the pulse do not change. The CTS frequency is identical to that of the laser. The CTS signal generated in the boosted frame can be transformed into the lab frame through

$$
a_{\mathrm{cts}}=\frac{e E_{\mathrm{cts}}}{m c \omega_{0}}=a_{\mathrm{cts}}^{\prime} D_{n l},
$$

where $a_{\mathrm{cts}}^{\prime}=e E_{\mathrm{cts}}^{\prime} / m c \omega^{\prime}=e E_{\mathrm{cts}} / m c \omega_{\mathrm{cts}}$.

Table I shows simulation parameters in both lab and boosted frames for the example given in Fig. 2. In the lab frame, the simulation of the high-frequency CTS signal requires ultrahigh resolution. In the boosted frame, both layer thickness and laser wavelength are of the same order of magnitude and can be easily handled. The charge separation field is also properly considered. For the CTS problem considered in this paper, the boosted PIC simulation is about 2 orders of magnitude faster than the conventional PIC simulation. The equivalence of both boosted and conventional PIC simulations has been verified.

\section{APPENDIX B: DRIFT COMPRESSION}

Here, we derive the RES compression ratio in the boosted frame. From Eqs. (15) and (16), one has the longitudinal velocity

$$
\beta_{x}^{\prime}=\frac{\left(\gamma_{0}^{\prime}+p_{0}^{\prime}\right)^{2}-\left(1+a^{2}\right)}{\left(\gamma_{0}^{\prime}+p_{0}^{\prime}\right)^{2}+\left(1+a^{2}\right)},
$$

where we have taken $a=a_{0} \tanh \left(\tau^{\prime} / T^{\prime}\right)$ and $\tau^{\prime}=$ $t^{\prime}+x^{\prime} / c$. Particle displacement is obtained from 


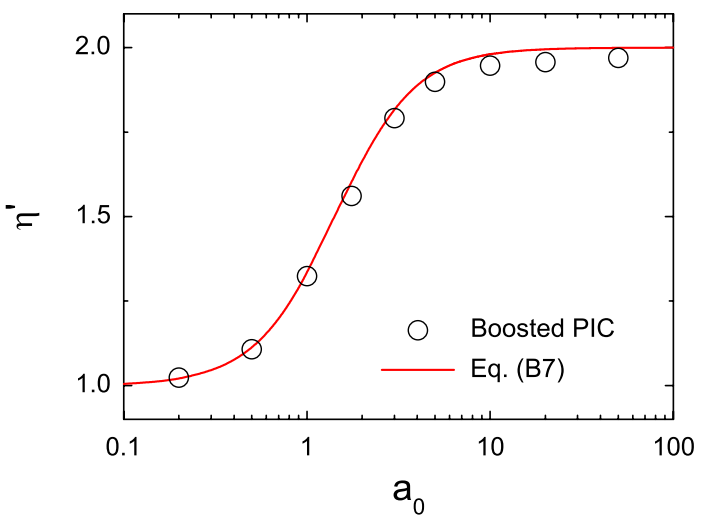

FIG. 7. Compression ratio in the boosted frame.

$$
x^{\prime}\left(\tau^{\prime}\right)=\int_{0}^{t^{\prime}} c \beta_{x}^{\prime} d t^{\prime \prime}=\int_{0}^{\tau^{\prime}} \frac{c \beta_{x}^{\prime}}{1+\beta_{x}^{\prime}} d \tau^{\prime \prime},
$$

where the relation $d \tau^{\prime} / d t^{\prime}=1+\beta_{x}^{\prime}$ has been used. Substituting Eq. (B1) into Eq. (B2), we get

$$
x^{\prime}\left(\tau^{\prime}\right)=\frac{a_{0}^{2} c T^{\prime}}{2\left(\gamma_{0}^{\prime}+p_{0}^{\prime}\right)^{2}} \tanh \left(\frac{\tau^{\prime}}{T^{\prime}}\right) .
$$

We consider two particles with initial positions $\left.x_{1}^{\prime}\right|_{t=0}=0$ and $\left.x_{2}^{\prime}\right|_{t=0}=-d x^{\prime}$. For particle one, position evolution is just given by Eq. (B3). For particle two, position evolution is

$$
x_{2}^{\prime}\left(\tau^{\prime}\right)=-\frac{d x^{\prime}}{1+\beta_{0}^{\prime}}+\frac{a_{0}^{2} c T^{\prime}}{2\left(\gamma_{0}^{\prime}+p_{0}^{\prime}\right)^{2}} \tanh \left(\frac{\tau^{\prime}}{T^{\prime}}\right),
$$

where $\beta_{0}^{\prime}=a_{0}^{2} /\left(2+a_{0}^{2}\right)$ [see Eq. (13)]. The final positions for both particles at rest are

$$
\begin{gathered}
\left.x_{1}^{\prime}\right|_{\tau^{\prime} \gg T^{\prime}}=\frac{a_{0}^{2} c T^{\prime}}{2\left(\gamma_{0}^{\prime}+p_{0}^{\prime}\right)^{2}}, \\
\left.x_{2}^{\prime}\right|_{\tau^{\prime} \gg T^{\prime}}=-\frac{d x^{\prime}}{1+\beta_{0}^{\prime}}+\frac{a_{0}^{2} c T^{\prime}}{2\left(\gamma_{0}^{\prime}+p_{0}^{\prime}\right)^{2}} .
\end{gathered}
$$

The compression ratio is

$$
\eta^{\prime}=\frac{d x^{\prime}}{\left.\left(x_{1}^{\prime}-x_{2}^{\prime}\right)\right|_{\tau^{\prime} \gg T}}=\frac{2+2 a_{0}^{2}}{2+a_{0}^{2}},
$$

which spans $\eta^{\prime} \in[1,2)$ for $a_{0}^{2} \in[0, \infty)$ as shown in Fig. 7 . From the above derivation, we find that $\eta^{\prime}$ is independent of any specific pulse shape. Therefore the explicit evaluation of Eq. (B2) is not necessary; only the term $-d x^{\prime} /\left(1+\beta_{0}^{\prime}\right)$ on the right side of Eq. (B4) is needed.

At the maximum laser amplitude $a_{0}$, we have the relation

$$
\left.d^{\prime}\right|_{a_{0}}=\left.\gamma_{b} d\right|_{a_{0}}
$$

for the thickness in both frames. When combined with Eq. (21), one obtains the compression ratio in the lab frame:

$$
\eta \equiv \frac{d_{0}}{\left.d\right|_{a_{0}}}=\eta^{\prime} \frac{\gamma_{0}^{\prime} \gamma_{b}}{\gamma_{0}}=\frac{\left(\gamma_{0}+p_{0}\right)^{2}+\left(1+a_{0}^{2}\right)}{2 \gamma_{0}\left(\gamma_{0}+p_{0}\right)} \gtrsim 1,
$$

which is generally very close to unity. With the help of Eqs. (9), (13), and (19), one recognizes that

$$
\begin{gathered}
\eta^{\prime}=\left.\gamma^{\prime}\right|_{a_{0}} / \gamma_{0}^{\prime}, \\
\eta=\left.\gamma\right|_{a_{0}} / \gamma_{0},
\end{gathered}
$$

which gives $\gamma_{0}^{\prime} d_{0}^{\prime}=\left.\left(\gamma^{\prime} d^{\prime}\right)\right|_{a_{0}}$ and $\gamma_{0} d_{0}=\left.(\gamma d)\right|_{a_{0}}$. Thus, $\gamma d$ or $n / \gamma$ are conserved quantities during RES compression in both the lab and the boosted frames.

[1] W. Ackermann et al., Nat. Photon. 1, 336 (2007).

[2] P. Emma et al., Nat. Photon. 4, 641 (2010).

[3] A. Einstein, Ann. Phys. (Leipzig) 322, 891 (1905).

[4] S. V. Bulanov, T.Zh. Esirkepov, and T. Tajima, Phys. Rev. Lett. 91, 085001 (2003).

[5] M. Kando et al., Phys. Rev. Lett. 99, 135001 (2007); A. S. Pirozhkov et al., Phys. Plasmas 14, 123106 (2007); M. Kando et al., Phys. Rev. Lett. 103, 235003 (2009).

[6] V. V. Kulagin, V. A. Cherepenin, M. S. Hur, and H. Suk, Phys. Rev. Lett. 99, 124801 (2007).

[7] J. Meyer-ter-Vehn and H.-C. Wu, Eur. Phys. J. D 55, 433 (2009).

[8] H.-C. Wu and J. Meyer-ter-Vehn, Eur. Phys. J. D 55, 443 (2009).

[9] H.-C. Wu, J. Meyer-ter-Vehn, J. Fernández, and B. M. Hegelich, Phys. Rev. Lett. 104, 234801 (2010).

[10] A. K. Geim and K.S. Novoselov, Nature Mater. 6, 183 (2007).

[11] S. Bae et al., Nature Nanotech. 5, 574 (2010).

[12] J. Bai, X. Zhong, S. Jiang, Y. Huang, and X. Duan, Nature Nanotech. 5, 190 (2010).

[13] G. Bressi, G. Carugno, R. Onofrio, and G. Ruoso, Phys. Rev. Lett. 88, 041804 (2002).

[14] A. Dubietis, R. Butkus, and A. P. Piskarskas, IEEE J. Sel. Top. Quantum Electron. 12, 163 (2006).

[15] D. Herrmann, L. Veisz, R. Tautz, F. Tavella, K. Schmid, V. Pervak, and F. Krausz, Opt. Lett. 34, 2459 (2009).

[16] L. Veisz (private communication).

[17] www.extreme-light-infrastructure.eu.

[18] H.-C. Wu, arXiv:1104.3163.

[19] P. M. Woodward, J. Inst. Electr. Eng. 93, 1554 (1947); J. D. Lawson, IEEE Trans. Nucl. Sci. 26, 4217 (1979).

[20] E. Esarey, P. Sprangle, and J. Krall, Phys. Rev. E 52, 5443 (1995).

[21] J.-L. Vay, Phys. Rev. Lett. 98, 130405 (2007).

[22] E. S. Sarachik and G. T. Schappert, Phys. Rev. D 1, 2738 (1970).

[23] J. Meyer-ter-Vehn, A. Pukhov, and Z.-M. Sheng, in Atoms, Solids and Plasmas in Super-Intense Laser Fields, edited by D. Batani et al. (Kluwer Academic/Plenum Publishers, New York, 2001). 